\title{
SECOND-ORDER CLOSURE MODELS FOR ROTATING TURBULENT FLOWS*
}

\author{
BY \\ CHARLES G. SPEZIALE \\ ICASE, NASA Langley Research Center \\ AND \\ Georgia Institute of Technologv, Atlanta, Georgia
}

\begin{abstract}
The physical properties of the commonly used second-order closure models are examined theoretically for rotating turbulent flows. Comparisons are made with results which are a rigorous consequence of the Navier-Stokes equations for the problem of fully-developed turbulent channel flow in a rapidly rotating framework. It is demonstrated that all existing second-order closures yield spurious physical results for this test problem of rotating channel flow. In fact, the results obtained are shown to be substantially more unphysical than those obtained from the simpler $K-\varepsilon$ and $K-l$ models. Modifications in the basic structure of these second-order closure models are proposed which can alleviate this problem.
\end{abstract}

1. Introduction. The ability to accurately predict rotating turbulent flows could have a wide variety of important applications ranging from the analysis of turbomachinery to the description of turbulence in the atmosphere or in oceans. However, with the exception of some recent work on the limiting case of two-dimensional turbulence [1-3], there appears to have been little work which accounts for the effects of rigid body rotations on turbulence modeling by a direct analysis of the Navier-Stokes equations. In fact, much of the work in modeling rotating flows has been conducted utilizing the $K-\varepsilon$ (or $K-l$ ) model of turbulence which requires the effects of rotations to be accounted for by the use of a variety of ad hoc empiricisms (cf. Majumdar, Pratap, and Spalding [4], Howard, Patankar, and Bordynuik [5], and Galmes and Lakshminarayana [6]). While such approaches can be useful in correlating experimental results for a particular class of rotating turbulent flows,

*Received October 7, 1986. 
they do not form the basis for a general theory which is needed if models are to be developed that truly have a predictive value.

The purpose of the present paper is to examine the consistency of various second-order closure models with results which are a rigorous consequence of the Navier-Stokes equations in a rapidly rotating framework. Although there have been a few previous studies of rotating turbulent flows using second-order closure models (cf. So [7], So and Peskin [8], and Mellor and Yamada [9]), only a narrow range of flows have been considered so that no definitive conclusions could be drawn about the correctness of the models. In this paper the test problem of fully-developed turbulent channel flow in a rapidly rotating framework will be considered. It will be proven, as a rigorous consequence of the Navier-Stokes equations, that the Reynolds stress tensor for this problem must be two-dimensional (as a direct consequence of the Taylor-Proudman theorem [10]) and must have a nonzero Reynolds shear stress in the plane of the flow. The commonly used second-order closure models yield completely opposite results (i.e., a three-dimensional Reynolds stress tensor with vanishing Reynolds shear stresses), and, hence, are fundamentally inconsistent with the Navier-Stokes equations for turbulent channel flow in a rapidly rotating framework. It is also demonstrated that the results obtained from these second-order closures are substantially more unphysical than those obtained from the simpler $K-\varepsilon$ or $K-l$ models. Modifications in existing second-order closure models which can alleviate this problem will be explored. By the addition of one term to the second-order closure obtained by Haworth and Pope [11] from a generalized Langevin model, it will be shown that consistency with the Navier-Stokes equations in a rapidly rotating frame can be achieved. Other possible modifications will be discussed briefly in the last section along with the prospects for future research.

2. Second-order closure models and rotating channel flow. The turbulent flow of a homogeneous and incompressible viscous fluid in a rotating frame of reference will be considered. The velocity field $\mathbf{v}$ and pressure $P$ will be decomposed into ensemble mean and fluctuating parts, respectively, as follows:

$$
\mathbf{v}=\overline{\mathbf{v}}+\mathbf{u}, \quad P=\bar{P}+p .
$$

Of course, the decompositions in Eq. (1) are solutions of the mean and fluctuating parts of the Navier-Stokes equations and continuity equation which, in a steadily rotating frame of reference, take the form [12]

$$
\begin{aligned}
\frac{\partial \overline{\mathbf{v}}}{\partial t}+\overline{\mathbf{v}} \cdot \nabla \overline{\mathbf{v}} & =-\nabla \bar{P}+\nu \nabla^{2} \overline{\mathbf{v}}-\nabla \cdot \tau-2 \Omega \times \overline{\mathbf{v}}, \\
\frac{\partial \mathbf{u}}{\partial t}+\overline{\mathbf{v}} \cdot \nabla \mathbf{u} & =-\mathbf{u} \cdot \nabla \mathbf{u}-\mathbf{u} \cdot \nabla \overline{\mathbf{v}}-\nabla p+\nu \nabla^{2} \mathbf{u}+\nabla \cdot \tau-2 \Omega \times \mathbf{u}, \\
\nabla \cdot \overline{\mathbf{v}} & =0, \\
\nabla \cdot \mathbf{u} & =0,
\end{aligned}
$$

where $\nu$ is the kinematic viscosity of the fluid, $\Omega$ is the angular velocity of the reference frame relative to an inertial framing, and $\tau$ is the kinematic Reynolds stress tensor given 
by

$$
\tau=\overline{\mathbf{u u}} \text {. }
$$

It should be noted that $\bar{P}$ in Eq. (2) is the modified mean pressure which includes the centrifugal and gravitational body force potentials.

The Reynolds stress transport equation is obtained by taking the ensemble mean of the symmetric part of the outer product of Eq. (3) with $\mathbf{u}$. This equation takes the form [12]

$$
\begin{aligned}
\frac{D \tau_{k l}}{D t}+\tau_{k m} \frac{\partial \bar{v}_{l}}{\partial x_{m}}+\tau_{l m} & \frac{\partial \bar{v}_{k}}{\partial x_{m}}=-\frac{\partial}{\partial x_{m}}\left(\overline{u_{m} u_{k} u_{l}}\right) \\
& -\overline{\left(u_{k} \frac{\partial p}{\partial x_{l}}+u_{l} \frac{\partial p}{\partial x_{k}}\right)}-2 \nu \overline{\frac{\partial u_{k}}{\partial x_{m}} \frac{\partial u_{l}}{\partial x_{m}}}+\nu \nabla^{2} \tau_{k l} \\
& +2 \varepsilon_{m k n} \Omega_{m} \tau_{n l}+2 \varepsilon_{m l n} \Omega_{m} \tau_{n k}
\end{aligned}
$$

in a rotating frame of reference. In (7), Cartesian tensor notation is utilized where the Einstein summation convention applies to repeated indices and $\varepsilon_{k l m}$ represents the permutation tensor. Second-order closure models are obtained when closure relations are provided that tie the higher-order turbulence correlations in (7) to the Reynolds stresses (along with their spatial gradients), the mean velocity gradients, and the length scale of turbulence. The closure relations used for the higher-order correlations (i.e., the triple velocity correlation, pressure gradient-velocity correlation, and the dissipation rate correlation) in an inertial framing are of the general form

$$
\begin{gathered}
\overline{u_{k} u_{l} u_{m}}=C_{k l m}(\tau, \nabla \tau, l), \\
\overline{u_{k} \frac{\partial p}{\partial x_{l}}+u_{l} \frac{\partial p}{\partial x_{k}}=}=-C_{1}\left(\tau_{k m} \frac{\partial \bar{v}_{l}}{\partial x_{m}}+\tau_{l m} \frac{\partial \bar{v}_{k}}{\partial x_{m}}\right)+A_{k l}(\tau, \nabla \tau, \overline{\mathbf{D}}, l), \\
2 \nu \frac{\partial u_{k}}{\partial x_{m}} \frac{\partial u_{l}}{\partial x_{m}}=B_{k l}(\tau, \overline{\mathbf{D}}, l),
\end{gathered}
$$

respectively (see Mellor and Herring [13], Launder, Reece, and Rodi [14], and Lumley [15]). In (7)-(10), $C_{1}$ is a dimensionless constant, $l$ is the length scale of turbulence which must be obtained from a separate transport equation, and

$$
\begin{aligned}
\frac{D}{D t} & =\frac{\partial}{\partial t}+\overline{\mathbf{v}} \cdot \nabla \\
\bar{D}_{k l} & =\frac{1}{2}\left(\frac{\partial \bar{v}_{k}}{\partial x_{l}}+\frac{\partial \bar{v}_{l}}{\partial x_{k}}\right)
\end{aligned}
$$

are, respectively, the mean substantial derivative and rate of strain tensor. By extending (8)-(10) to rotating frames of reference (see Speziale [2]) and then substituting the results into (7), it is a simple matter to show that all existing second-order closure models are of the form

$$
\begin{aligned}
\frac{D \tau_{k l}}{D t}+\left(1-C_{1}\right)\left(\tau_{k m} \frac{\partial \bar{v}_{l}}{\partial x_{m}}\right. & \left.+\tau_{l m} \frac{\partial \bar{v}_{k}}{\partial x_{m}}\right)=-\frac{\partial}{\partial x_{m}} C_{m k l}(\tau, \nabla \tau, l) \\
& -A_{k l}(\tau, \nabla \tau, \overline{\mathbf{D}}, l)-B_{k l}(\tau, \overline{\mathbf{D}}, l)+\nu \nabla^{2} \tau_{k l} \\
& +\left(2-C_{1}\right)\left(\varepsilon_{m k n} \Omega_{m} \tau_{n l}+\varepsilon_{m l n} \Omega_{m} \tau_{n k}\right)
\end{aligned}
$$


in a rotating frame of reference. It should be noted that in the Rotta-Kolmogorov model [13] the constant $C_{1}=0$ and in the Launder, Reece, and Rodi [14] and Lumley [15] models, $C_{1}$ is a nonzero constant which does not equal 2. Hence, the Coriolis term on the right-hand side of (13) survives in all existing second-order closures where it constitutes the only noninertial term.

Now, we will consider the test problem of fully-developed turbulent channel flow in a rapidly rotating framework (see Fig. 1). This fully-developed channel flow is maintained by a constant axial mean pressure gradient,

$$
\frac{\partial \bar{P}}{\partial x}=-G
$$

while the channel is subjected to a steady spanwise rotation with the angular velocity (see Fig. 1)

$$
\Omega=\Omega \mathbf{k}
$$

The mean velocity field is given by

$$
\overline{\mathbf{v}}=\bar{U}(y) \mathbf{i}
$$

and the Reynolds stress tensor is of the form

$$
\tau=\tau(y)
$$

since the flow conditions are fully-developed. Hence, the axial component of the Reynolds equation (2) in a rotating channel flow is given by

$$
\nu \frac{d^{2} \bar{U}}{d y^{2}}-\frac{d \tau_{x y}}{d y}+G=0
$$
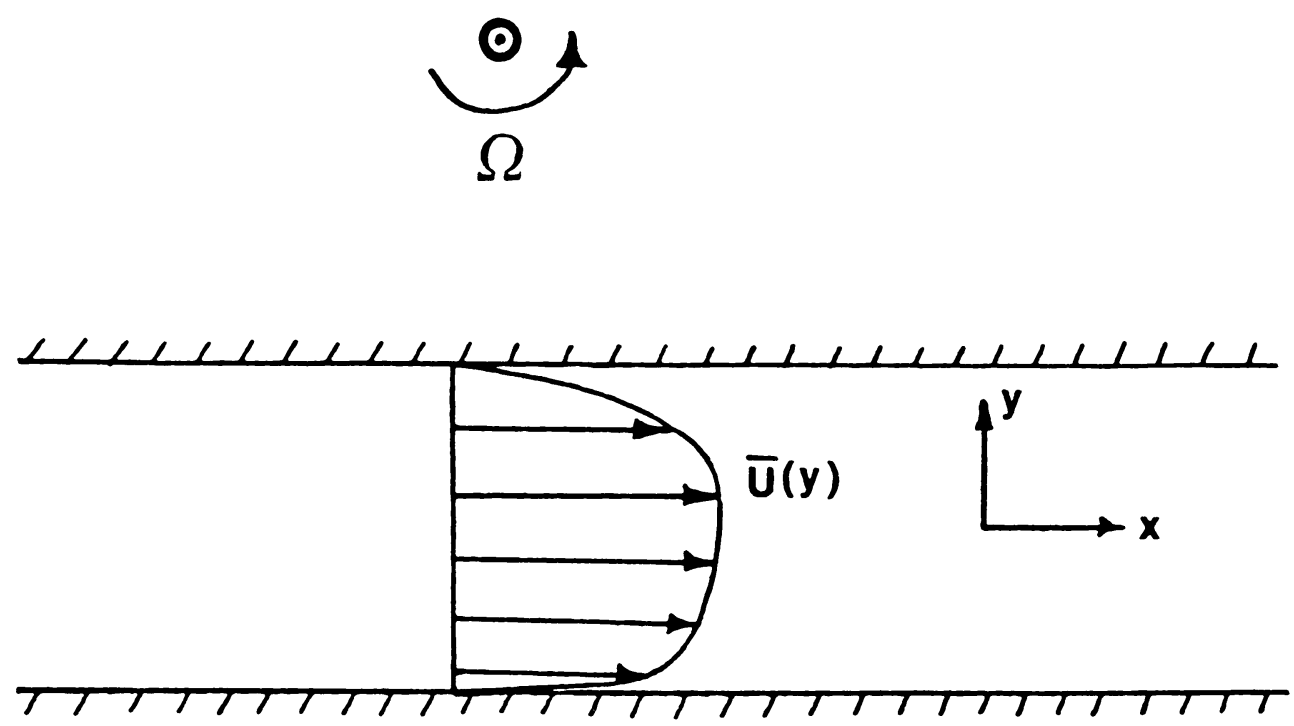

FIG. 1. Fully-Developed Turbulent Channel Flow Subjected to a Spanwise Rotation after Johnston, Halleen, and Lezius [16]. 
Since it is well known that the axial velocity profile in fully-developed turbulent channel flow is not parabolic like its laminar counterpart, it is clear that

$$
\tau_{x y} \neq 0
$$

(also see the experimental results of Johnston, Halleen, and Lezius [16]). The $y$ - and $z$-components of the Reynolds equation (2) take the form

$$
\begin{gathered}
-\frac{\partial \bar{P}}{\partial y}-\frac{d \tau_{y y}}{d y}-2 \Omega \bar{U}=0, \\
-\frac{\partial \bar{P}}{\partial z}-\frac{d \tau_{y z}}{d y}=0 .
\end{gathered}
$$

Since there is no forcing in the $z$-direction,

$$
\frac{\partial \bar{P}}{\partial z}=0
$$

and (21) can be integrated to yield the result

$$
\tau_{y z}=0
$$

since $\tau$ must satisfy the no slip condition at the channel walls. Similarly, it can be shown that

$$
\tau_{x z}=0
$$

The vorticity transport equation in a steadily rotating framework (where $\omega=\nabla \times \mathbf{v}$ ) takes the form [10]

$$
\frac{D \omega}{D t}=\omega \cdot \nabla \mathbf{v}+\nu \nabla^{2} \boldsymbol{\omega}+2 \Omega \cdot \nabla \mathbf{v}
$$

A rapidly rotating framework is defined as one in which

$$
\Omega t_{0} \gg 1
$$

where $t_{0}$ is the time scale of the turbulent fluctuations. For a rotating channel flow where $\boldsymbol{\Omega}=\boldsymbol{\Omega} \mathbf{k},(24)$ can be written in the equivalent form

$$
\frac{1}{\Omega} \frac{D \omega}{D t}=\frac{1}{\Omega} \omega \cdot \nabla \mathbf{v}+\frac{\nu}{\Omega} \nabla^{2} \omega+2 \mathbf{k} \cdot \nabla \mathbf{v} .
$$

As a result of (25), equation (24) yields the approximate constraint (in a rapidly rotating framework) that

$$
2 \mathbf{k} \cdot \nabla \mathbf{v}=0
$$

It, thus, follows that

$$
\frac{\partial \mathbf{v}}{\partial z}=0
$$

Since the walls of the channel are parallel to the axis of rotation, (28) has the exact solution

$$
\mathbf{v}=\mathbf{v}(x, y, t)
$$


which is valid throughout the channel. Hence, the flow must become two-dimensional (in a strong approximate sense) as would be expected from the Taylor-Proudman theorem [10]. Since $\overline{\mathbf{v}}=\bar{U}(y) \mathbf{i}$, it is clear that the fluctuating velocity is of the approximate form

$$
\mathbf{u}=\mathbf{u}(x, y, t)
$$

in a rapidly rotating channel flow. It will now be shown that, since there is no mean forcing along the axis of rotation (i.e., since $\bar{v}_{z}=\partial \bar{P} / \partial z=0$ ), it follows that

$$
u_{z}=0 \text {. }
$$

This result can be easily seen by examining the $z$-component of the fluctuating momentum equation (3) which reduces to the form

$$
\frac{\partial u_{z}}{\partial t}+\bar{U} \frac{\partial u_{z}}{\partial x}=-u_{x} \frac{\partial u_{z}}{\partial x}-u_{y} \frac{\partial u_{z}}{\partial y}+\nu \nabla^{2} u_{z}
$$

since $\partial p / \partial z=0$ (the flow is unforced in the $z$-direction). Once $\bar{U}, u_{x}$, and $u_{y}$ are determined from the $x$ - and $y$-components of the momentum equations (2) and (3), equation (32) constitutes a linear partial differential equation for the determination of $u_{z}$. This equation has the simple solution $u_{z}=0$ when solved subject to the no slip condition at the channel walls. Hence, the solution

$$
\begin{aligned}
\tau_{x z} & =\overline{u_{x} u_{z}}=0, \\
\tau_{z z} & =\overline{u_{z} u_{z}}=0
\end{aligned}
$$

is consistent with the Navier-Stokes equations. It is thus clear that, as a rigorous consequence of the Navier-Stokes equations, the Reynolds stress tensor in a rapidly rotating channel flow takes the two-dimensional form

$$
\tau=\left[\begin{array}{ccc}
\tau_{x x}(y) & \tau_{x y}(y) & 0 \\
\tau_{x y}(y) & \tau_{y y}(y) & 0 \\
0 & 0 & 0
\end{array}\right] .
$$

This differs from $\tau$ for turbulent channel flow in an inertial framework only in that $\tau_{z z}$ is zero-a result which is a direct consequence of the Taylor-Proudman reorganization that occurs in a rapidly rotating framework.

It will now be demonstrated that all existing second-order closure models yield results that are fundamentally inconsistent with (35) and, hence, are in serious violation of the Navier-Stokes equations for rotating channel flow. By establishing a one-to-one correspondence between $(x, y, z)$ and $\left(x_{1}, x_{2}, x_{3}\right)$, the general form of second-order closures given by (13) reduces to the equation

$$
\begin{aligned}
\frac{1}{\Omega}\left[\frac{D \tau_{k l}}{D t}+\left(1-C_{1}\right)\right. & \left.\left(\tau_{k m} \frac{\partial \bar{v}_{l}}{\partial x_{m}}+\tau_{l m} \frac{\partial \bar{v}_{k}}{\partial x_{m}}\right)\right]=-\frac{1}{\Omega} \frac{\partial C_{m k l}}{\partial x_{m}}(\tau, \nabla \tau, l) \\
& -\frac{1}{\Omega} A_{k l}(\tau, \nabla \tau, \overline{\mathbf{D}}, l)-\frac{1}{\Omega} B_{k l}(\tau, \overline{\mathbf{D}}, l)+\frac{\nu}{\Omega} \nabla^{2} \tau_{k l} \\
& +\left(2-C_{1}\right)\left(\varepsilon_{3 k n} \tau_{n l}+\varepsilon_{3 l n} \tau_{n k}\right)
\end{aligned}
$$


where we have made use of the fact that $\Omega=\Omega \mathbf{k}$ and we have divided through by $\Omega$. Taking the limit of (36) as $\Omega \rightarrow \infty$, we conclude that all existing second-order closure models yield the constraint

$$
\left(2-C_{1}\right)\left(\varepsilon_{3 k n} \tau_{n l}+\varepsilon_{3 l n} \tau_{n k}\right)=0
$$

for a rapidly rotating channel flow. As noted earlier,

$$
2-C_{1} \neq 0
$$

for all of the existing second-order closures (in fact, $C_{1}=0$ for the Rotta-Kolmogorov model and $C_{1}<1$ in the Launder models [14]). It is then a simple matter to show that (37) has the general solution

$$
\begin{aligned}
\tau_{x x} & =\tau_{y y}, \\
\tau_{x y} & =0 .
\end{aligned}
$$

Hence, all existing second-order closures predict a state of transverse isotropy for turbulent channel flow in a rapidly rotating framework - a result which is in conflict with the Navier-Stokes equations as demonstrated earlier. In fact, this constitutes a completely spurious physical result since it is well known that a turbulent shear flow must be accompanied by a nonzero turbulent shear stress. Furthermore, since the Coriolis term vanishes in the $z z$-component of all existing second-order closures (see Eq. (13)), it is clear that these models predict that

$$
\tau_{z z} \neq 0
$$

as they do for a turbulent channel flow in an inertial framing. This result is inconsistent with the Taylor-Proudman theorem for rotating channel flow as discussed earlier. These inconsistencies arise because all existing second-order closure models violate the principle of material frame-indifference in the limit of two-dimensional turbulence-a result which is a rigorous consequence of the Navier-Stokes equations as proven by Speziale [1, 17]. To be more specific, the inertial terms in (13) do not vanish in a two-dimensional turbulence unless it is isotropic. In the next section, it will be shown how these deficiencies can be remedied.

3. Alternative turbulence models for rotating flows. As a prelude to examining alterations in existing second-order closure models which can remedy the deficiencies discussed in the previous section, we will consider the consistency of the simpler $K-\varepsilon$ or $K-l$ models of turbulence with the Navier-Stokes equations for rotating channel flow. In the $K-\varepsilon$ or $K-l$ models of turbulence, the Reynolds stress tensor takes the form

$$
\tau_{k l}=\frac{2}{3} K \delta_{k l}-\alpha K^{1 / 2} l\left(\frac{\partial \bar{v}_{k}}{\partial x_{l}}+\frac{\partial \bar{v}_{l}}{\partial x_{k}}\right),
$$

where

$$
K=\frac{1}{2} \tau_{m m}
$$

is the turbulent kinetic energy per unit mass which is obtained from a modeled version of its transport equation, $l$ is the length scale of turbulence, and $\alpha$ is a dimensionless constant. For the $K-l$ model of turbulence, $l$ is either specified algebraically or is 
determined from a modeled transport equation obtained from the contracted form of the evolution equation for the two-point velocity correlations [13]. In the $K-\varepsilon$ model, the length scale is given by

$$
l=\frac{K^{3 / 2}}{\varepsilon}
$$

where $\varepsilon$ is the dissipation rate of the turbulence which is obtained from a modeled version of its transport equation [14]. Since the tensorial dependence of (42) is only on the Kronecker delta and the symmetric part of the mean velocity gradients which are frame-indifferent [20], these traditional $K-\varepsilon$ or $K-l$ models are of the same form in a rotating frame as in an inertial framing. The only way that the effects of rotation are accounted for is through a change in the length scale which is usually done with ad hoc empiricisms (see Howard, Patankar, and Bordynuik [5]). This result (along with the fact that the mean flow is unidirectional so that the Coriolis acceleration in (2) affects only the mean pressure [17]) guarantees that the basic structure of the Reynolds stress tensor predicted by the $K-\varepsilon$ or $K-l$ models will be the same in an inertial or a rotating frame of reference. To be more specific, the Reynolds stress tensor obtained from $K-\varepsilon$ or $K-l$ models of turbulence will be of the form

$$
\boldsymbol{\tau}=\left[\begin{array}{ccc}
\tau_{x x} & \tau_{x y} & 0 \\
\tau_{x y} & \tau_{y y} & 0 \\
0 & 0 & \tau_{z z}
\end{array}\right]
$$

(where $\tau_{x x}=\tau_{y y}=\tau_{z z}$ ) for rotating channel flow or for channel flow in an inertial framing. Of course, the specific values of the nonvanishing components of $\tau$ can be different in the rotating frame as a result of changes in the length scale. While (45) is not correct for a turbulent channel flow in a rapidly rotating framework (since $\tau_{z z} \neq 0$ ), it is substantially less unphysical than results obtained from existing second-order closures where $\tau_{x y}$ is zero.

More recently, Galmes and Lakshminarayana [6] developed an algebraic model for rotating flows which is given by

$$
\begin{aligned}
\tau_{k l}=\frac{2}{3} K \delta_{k l}-\frac{2 K}{C_{1} \varepsilon}\left(1-\frac{1}{2} C_{2}\right)\left(\varepsilon_{k m n} \Omega_{m} \tau_{n l}+\varepsilon_{l m n} \Omega_{m l} \tau_{n k}\right) \\
+\frac{K\left(1-C_{2}\right)}{C_{1} \varepsilon}\left[\frac{2}{3} \tau_{m n} \frac{\partial \bar{v}_{m}}{\partial x_{n}} \delta_{k l}-\left(\tau_{k m} \frac{\partial \bar{v}_{l}}{\partial x_{m}}+\tau_{l m} \frac{\partial \bar{v}_{k}}{\partial x_{m}}\right)\right]
\end{aligned}
$$

where $C_{1}$ and $C_{2}$ are dimensionless constants that take on the approximate values of 1.5 and 0.6 , respectively. For rotating channel flow where

$$
\Omega=\Omega \mathbf{k}
$$

Eq. (46) can be written in the equivalent form

$$
\begin{aligned}
& \frac{1}{\Omega} \tau_{k l}=\frac{2}{3} \frac{K}{\Omega} \delta_{k l}+\frac{2 K}{C_{1} \varepsilon}\left(1-\frac{1}{2} C_{2}\right)\left(\varepsilon_{3 k n n l} \tau_{n / n} \tau_{n k}\right) \\
&+\frac{K\left(1-C_{2}\right)}{\Omega C_{1} \varepsilon}\left[\frac{2}{3} \tau_{m n} \frac{\partial \bar{v}_{m}}{\partial x_{n}} \delta_{k l}-\left(\tau_{k m} \frac{\partial \bar{v}_{l}}{\partial x_{m}}+\tau_{l m} \frac{\partial \bar{v}_{k}}{\partial x_{m}}\right)\right]
\end{aligned}
$$


which, in a rapidly rotating framework (i.e., for $\Omega \rightarrow \infty$ ), yields the approximate constraint

$$
\varepsilon_{3 k n} \tau_{n l}+\varepsilon_{3 / n} \tau_{n k}=0 .
$$

Equation (48) requires that $\tau_{x x}=\tau_{y y}$ and $\tau_{x y}=0$. Hence, the algebraic Reynolds stress model for rotating flows proposed by Galmes and Lakshminarayana [6] yields the same spurious result for a rapidly rotating channel flow (i.e., a vanishing Reynolds shear stress in a turbulent shear flow) as that predicted by existing second-order closure models. This problem arises since the inertial term in (47) does not vanish for a two-dimensional turbulence and, hence, (47) is in violation of the principle of material frame-indifference which is a rigorous consequence of the Navier-Stokes equations in the two-dimensional limit.

It will now be demonstrated that these problems can be overcome by making a small modification in the second-order closure recently obtained by Haworth and Pope [11] from a generalized Langevin model. In this Langevin model, a Reynolds stress closure is obtained which, in an inertial framing, takes the form

$$
\frac{D \tau_{k l}}{D t}+\tau_{k m} \frac{\partial \bar{v}_{l}}{\partial x_{m}}+\tau_{l m} \frac{\partial \bar{v}_{k}}{\partial x_{m}}=-\frac{\partial}{\partial x_{m}}\left(\overline{u_{m} u_{k} u_{l}}\right)+G_{k m} \tau_{m l}+G_{l m} \tau_{m k}+C_{0} \varepsilon \delta_{k l}
$$

where $C_{0}$ is a dimensionless constant and $\mathbf{G}$ is a second rank tensor which is assumed to be of the general functional form

$$
\mathbf{G}=\mathbf{G}(\tau, \nabla \overline{\mathbf{v}}, \varepsilon)
$$

It should be noted that in this Langevin model it is not necessary to model the triple velocity correlation $\overline{\mathbf{u u u}}$; this term is closed since it arises from the convection in physical space of the joint probability density function which is obtained from a separate transport equation. Haworth and Pope [11] arrived at a model for $\mathbf{G}$ of the form

$$
G_{k l}=\left(2+\beta_{1}\right) \frac{\partial \bar{v}_{k}}{\partial x_{l}}-\frac{1}{2 K} \beta_{1} \frac{\partial \bar{v}_{k}}{\partial x_{m}} \tau_{m l}+H_{k l}(\tau, \overline{\mathbf{D}}, \varepsilon)
$$

where $\beta_{1}$ is a dimensionless constant and $\mathbf{H}$ is a frame-indifferent function since it depends only on the frame-indifferent quantities $\tau, \overline{\mathbf{D}}$, and $\varepsilon$. Hence the Langevin model (49) is, in an inertial framing, given by the equation

$$
\begin{aligned}
\frac{D \tau_{k l}}{D t}-\tau_{k m} \frac{\partial \bar{v}_{l}}{\partial x_{m}}-\tau_{l m} \frac{\partial \bar{v}_{k}}{\partial x_{m}}=-\frac{\partial}{\partial x_{m}}\left(\overline{u_{m} u_{k} u_{l}}\right) & \\
+\beta_{1}\left(\tau_{k m} \frac{\partial \bar{v}_{l}}{\partial x_{m}}+\tau_{l m} \frac{\partial \bar{v}_{k}}{\partial x_{m}}\right)-\frac{\beta_{1}}{2 K}\left(\frac{\partial \bar{v}_{k}}{\partial x_{m}} \tau_{m n} \tau_{n l}+\frac{\partial \bar{v}_{l}}{\partial x_{m}} \tau_{m n} \tau_{n k}\right) & +H_{k m} \tau_{m l}+H_{l m} \tau_{m k}+C_{0} \varepsilon \delta_{k l}
\end{aligned}
$$


where the reader is referred to Haworth and Pope [11] for the precise form of $\mathbf{H}$. It is a simple matter to show that in a rotating framework (with angular velocity $\Omega=\Omega \mathbf{k}$ ), Eq. (52) is given by

$$
\begin{aligned}
\frac{D \tau_{k l}}{D t}-\tau_{k m} \frac{\partial \bar{v}_{l}}{\partial x_{m}}-\tau_{l m} \frac{\partial \bar{v}_{k}}{\partial x_{m}} & =-\frac{\partial}{\partial x_{m}}\left(\overline{u_{m} u_{k} u_{l}}\right) \\
+ & \beta_{1}\left(\tau_{k m} \frac{\partial \bar{v}_{l}}{\partial x_{m}}+\tau_{l m} \frac{\partial \bar{v}_{k}}{\partial x_{m}}\right)-\frac{\beta_{1}}{2 K}\left(\frac{\partial \bar{v}_{k}}{\partial x_{m}} \tau_{m n} \tau_{n l}+\frac{\partial \bar{v}_{l}}{\partial x_{m}} \tau_{m n} \tau_{n k}\right) \\
+ & H_{k m} \tau_{m l}+H_{l m} \tau_{m k}+C_{0} \varepsilon \delta_{k l} \\
& -\beta_{1} \Omega\left[\varepsilon_{3 l m} \tau_{k m}+\varepsilon_{3 k m} \tau_{l m}-\frac{1}{2 K}\left(\varepsilon_{3 k m} \tau_{m n} \tau_{n l}+\varepsilon_{3 l m} \tau_{m n} \tau_{n k}\right)\right] .
\end{aligned}
$$

Haworth and Pope [11] proved that the inertial term in (53) vanishes for a two-dimensional turbulence and, hence, this Langevin model satisfies the principle of material frame-indifference in the two-dimensional limit unlike all other existing second-order closures. However, these authors also showed that this model does not give rise to a Taylor-Proudman reorganization in a rapidly rotating framework. In fact, it can be shown that for a turbulent channel flow in a rapidly rotating framework, (53) yields a stress tensor of the form (45). While this is a substantial improvement over what is predicted by all other existing second-order closures, it is still not completely correct in that $\tau_{z z}$ should vanish in the limit as $\Omega \rightarrow \infty$.

It will now be demonstrated that by making a small modification in the model for the tensor $\mathbf{G}$ proposed by Haworth and Pope [11], a second-order closure can be obtained that gives rise to a Taylor-Proudman reorganization in rapidly rotating turbulent flows, thus alleviating the problems discussed above. This proposed modification to (51) is as follows:

$$
\begin{aligned}
G_{k l}= & \frac{\beta_{2}}{K^{1 / 2}}\left|\bar{W}_{m n} \bar{W}_{n p} \tau_{p m}-K \bar{W}_{m n} \bar{W}_{n m}\right|^{1 / 2} \delta_{k l} \\
& +\left(2+\beta_{1}\right) \frac{\partial \bar{v}_{k}}{\partial x_{l}}-\frac{1}{2 K} \beta_{1} \frac{\partial \bar{v}_{k}}{\partial x_{m}} \tau_{m l}+H_{k l}(\tau, \overline{\mathbf{D}}, \varepsilon)
\end{aligned}
$$

where

$$
\bar{W}_{k l}=\frac{1}{2}\left(\frac{\partial \bar{v}_{k}}{\partial x_{l}}-\frac{\partial \bar{v}_{l}}{\partial x_{k}}\right)
$$

and $\beta_{2}$ is a dimensionless constant (for $\beta_{2}=0$, this model reduces to that of Haworth and Pope). Since the new term containing the coefficient $\beta_{2}$ vanishes in a two-dimensional turbulence, it follows that this model also satisfies the principle of material frame-indifference in the limit of two-dimensional turbulence. In a rotating framework with an angular velocity $\boldsymbol{\Omega}=\Omega \mathbf{k}$, this modified expression for $\mathbf{G}$ gives rise to a Langevin model of 
the form

$$
\begin{aligned}
\frac{D \tau_{k l}}{D t}-\tau_{k m} \frac{\partial \bar{v}_{l}}{\partial x_{m}}-\tau_{l m} \frac{\partial \bar{v}_{k}}{\partial x_{m}}= & -\frac{\partial}{\partial x_{m}}\left(\overline{u_{m} u_{k} u_{l}}\right) \\
& +\beta_{1}\left(\tau_{k m} \frac{\partial \bar{v}_{l}}{\partial x_{m}}+\tau_{l m} \frac{\partial \bar{v}_{k}}{\partial x_{m}}\right)-\frac{\beta_{1}}{2 K}\left(\frac{\partial \bar{v}_{k}}{\partial x_{m}} \tau_{m n} \tau_{n l}+\frac{\partial \bar{v}_{l}}{\partial x_{m}} \tau_{m n} \tau_{n k}\right) \\
& +H_{k m} \tau_{m l}+H_{l m} \tau_{m k}+C_{0} \varepsilon \delta_{k l} \\
& -\beta_{1} \Omega\left[\varepsilon_{3 l m} \tau_{k m}+\varepsilon_{3 k m} \tau_{m l}-\frac{1}{2 K}\left(\varepsilon_{3 k m} \tau_{m n} \tau_{n l}+\varepsilon_{3 l m} \tau_{m n} \tau_{n k}\right)\right] \\
& +\frac{2 \beta_{2}}{K^{1 / 2}} \mid\left(\bar{W}_{m n}-\varepsilon_{3 m n} \Omega\right)\left(\bar{W}_{n p}-\varepsilon_{3 n p} \Omega\right) \tau_{p m} \\
& -\left.K\left(\bar{W}_{m n}-\varepsilon_{3 m n} \Omega\right)\left(\bar{W}_{n m}-\varepsilon_{3 n m} \Omega\right)\right|^{1 / 2} \tau_{k l} .
\end{aligned}
$$

If we consider the case of a rapidly rotating framework (i.e., the limit as $\Omega \rightarrow \infty$ ), Eq. (56) yields the constraint

$$
\begin{aligned}
-\beta_{1}\left[\varepsilon_{3 / m} \tau_{k m}+\varepsilon_{3 k m} \tau_{l m}-\frac{1}{2 K}\left(\varepsilon_{3 k m} \tau_{m n} \tau_{n l}\right.\right. & \left.\left.+\varepsilon_{3 l m} \tau_{m n} \tau_{n k}\right)\right] \\
& +\frac{2 \beta_{2}}{K^{1 / 2}}\left|\varepsilon_{3 m n} \varepsilon_{3 n p} \tau_{p m}-K \varepsilon_{3 m n} \varepsilon_{3 n m}\right|^{1 / 2} \tau_{k l}=0 .
\end{aligned}
$$

A straightforward, although somewhat tedious, calculation shows that (57) is satisfied if and only if the Reynolds stress tensor takes the two-dimensional form

$$
\tau=\left[\begin{array}{ccc}
\tau_{x x} & \tau_{x y} & 0 \\
\tau_{x y} & \tau_{y y} & 0 \\
0 & 0 & 0
\end{array}\right]
$$

The key result that $\tau_{z z}$ must be zero can easily be seen by examining the $z z$-component of (57) which reduces to

$$
\frac{2 \beta_{2}}{K^{1 / 2}} \tau_{z z}^{3 / 2}=0
$$

It is thus clear that with just one minor modification, the Langevin second-order closure of Haworth and Pope yields a turbulence model that is consistent with the Taylor-Proudman theorem and is, thus, suitable for the description of rapidly rotating turbulent flows unlike all other existing turbulence models.

4. Conclusion. It has been proven that all existing second-order closure models are fundamentally inconsistent with the Navier-Stokes equations in a rapidly rotating framework. In particular, it was demonstrated that, for the test problem of fully-developed

${ }^{1}$ No constraint is placed on $\tau_{x x}, \tau_{x y}$, and $\tau_{y y}$ since Eq. (57) automatically vanishes for a two-dimensional turbulence. 
turbulent channel flow subjected to a rapid spanwise rotation, all existing second-order closures yield the spurious physical result of a vanishing Reynolds shear stress and are inconsistent with the Taylor-Proudman theorem. The type of results predicted for this problem by these second-order closures are, in fact, substantially more unphysical than those obtained from the simpler $K-\varepsilon$ or $K-l$ models of turbulence as indicated in Sec. 3. By adding one term to the second-order closure of Haworth and Pope obtained from a Langevin model, it was demonstrated that these problems can be overcome. This modified Langevin model appears to yield the first Reynolds stress closure that is generally consistent with the Navier-Stokes equations in a rapidly rotating framework.

Future calculations should be conducted on rotating turbulent flows with this modified Langevin model. This is quite important since the closure model for $\mathbf{G}$ given in (54) is not the most general and, hence, there may be other terms which could have been overlooked. Unfortunately, such a computational study is quite involved and is beyond the scope of the present paper. Future research is also needed on the effect of rotations on the evolution of the joint probability density function since such evolution equations play an important role in all Langevin models. Few, if any, studies along these lines appear to have been reported.

With some additional refinements, turbulent closure models can be developed that apply to rotating flows for a wide range of rotation rates without the need for ad hoc empiricisms. Such a development could have a profound effect on the analysis of a wide variety of turbulent flows that are of interest to engineers and geophysicists.

Acknowledgments. The author would like to thank Dr. T. B. Gatski of the NASA Langley Research Center for some valuable comments. This research was supported under NASA Contract No. NAS1-17070 while the author was in residence at the Institute for Computer Applications in Science and Engineering, NASA Langley Research Center, Hampton, VA 23665.

\section{REFERENCES}

[1] C. Speziale, Some interesting properties of two-dimensional turbulence, Phys. Fluids 24, 1425 (1981)

[2] C. Speziale, Closure models for rotating two-dimensional turbulence, Geophys. Astrophys. Fluid Dyn. 23, 69 (1983)

[3] C. Speziale, Modeling the pressure gradient-velocity correlation of turbulence, Phys. Fluids 28, 69 (1985)

[4] A. K. Majumdar, V. S. Pratap, and D. B. Spalding, Numerical computation of flow in rotating ducts, ASME J. Fluids Eng. 99. 148 (1977)

[5] J. H. Howard, S. V. Patankar, R. M. Bordynuik, Flow prediction in rotating ducts using Coriolis-modified turbulence models, ASME J. Fluids Eng. 102, 456 (1980)

[6] J. M. Galmes and B. Lakshminarayana, A turbulence model for three-dimensional turbulent shear flows over curved rotating bodies, AIAA Paper 83-0559 (1983)

[7] R. M. C. So, A turbulent velocity scale for curved shear flows, J. Fluid Mech. 70, 37 (1975)

[8] R. M. C. So and R. L. Peskin, Comments on extended pressure-strain correlation models, ZAMP 31, 56 (1980)

[9] G. L. Mellor and T. Yamada, A hierarchy of turbulence closure models for planetary boundary lavers, J. Atmos. Sci. 31, 1791 (1974)

[10] H. Greenspan, The Theory of Rotating Fluids, Cambridge Univ. Press, New York, 1968

[11] D. C. Haworth and S. B. Pope, A generalized Langevin model for turbulent flows, Phys. Fluids 29, 387 (1986)

[12] J. O. Hinze, Turbulence, McGraw-Hill, New York, 1975

[13] G. L. Mellor and H. J. Herring, A survey of the mean turbulent field closure models, AIAA J. 11, 590 (1973) 
[14] B. E. Launder, G. Reece, and W. Rodi, Progress in the development of a Reynolds-stress turbulence closure, J. Fluid Mech. 68, 537 (1975)

[15] J. L. Lumley, Turbulence Modeling, Adv. Appl. Mech. 18, 123 (1978)

[16] J. P. Johnston, R. M. Halleen, and D. K. Lezius, Effects of spanwise rotation on the structure of two-dimensional fully developed turbulent channel flow, J. Fluid Mech. 56, 533 [16].

[17] C. Speziale, Material frame-indifference in turbulence modeling, ASME J. Appl. Mech. 51, 942 (1984) 